\title{
BMJ Open Association between diabetes, metabolic syndrome and heart attack in US adults: a cross-sectional analysis using the Behavioral Risk Factor Surveillance System 2015
}

Guang-Ran Yang, ${ }^{\oplus 1,2}$ Timothy D Dye, ${ }^{2}$ Dongmei $\mathrm{Li}^{2}$

To cite: Yang G-R, Dye TD Li D. Association between diabetes, metabolic syndrome and heart attack in US adults: a cross-sectional analysis using the Behavioral Risk Factor Surveillance System 2015. BMJ Open 2019;9:e022990. doi:10.1136/ bmjopen-2018-022990

- Prepublication history for this paper is available online. To view these files, please visit the journal online (http://dx.doi.org/ 10.1136bmjopen-2018-022990)

Received 25 March 2018 Revised 15 July 2019 Accepted 30 August 2019

A) Check for updates

(c) Author(s) (or their employer(s)) 2019. Re-use permitted under CC BY-NC. No commercial re-use. See rights and permissions. Published by BMJ.

${ }^{1}$ Department of Endocrinology, Beijing Tongren Hospital, Capital Medical University, Beijing,

China

${ }^{2}$ Clinical and Translational Science Institute, School of Medicine and Dentistry, University of Rochester, Rochester, New York, USA

Correspondence to Dr Guang-Ran Yang; gr.yang@ccmu.edu.cn

Professor Dongmei Li; Dongmei_li@URMC.rochester. edu

\section{ABSTRACT}

Objectives Diabetes mellitus (DM) and metabolic syndrome (MS) are both associated with heart attack. Evidence regarding which condition-MS or DM-is better associated with heart attack, however, is limited. The purpose of this study is to examine DM and MS, and their comparative associations with heart attack, using the 2015 Behavioral Risk Factor Surveillance System (BRFSS). Design Cross-sectional study.

Methods A total of 332008 subjects aged over 18 years were included in the analysis. All subjects were classified into four groups based on their DM and MS status: neither DM nor MS, DM without MS, MS without DM, and both DM and MS. A weighted hierarchical logistic regression was used to examine the difference between the four groups in their association with the risk of a heart attack.

Results Differences in weighted frequency distributions of gender, age category (over 45 years or not), smoking status, education, race, physical activity and daily vegetable and fruit consumption were significantly different across the four groups $(p<0.05)$. The weighted prevalence of heart attack was $5.2 \%$ for neither DM nor MS group, $8.5 \%$ for DM without MS group, $11.0 \%$ for MS without DM group and $16.1 \%$ for both DM and MS group. The weighted prevalence of heart attack in MS without DM group was significantly higher than that in the DM without MS group $(p<0.01)$. After adjusting for confounding variables, DM without MS and MS without DM were both found to be independently associated with heart attack compared with those without DM nor MS (DM without MS, $\mathrm{OR}=2.09$; $\mathrm{MS}$ without $\mathrm{DM}, \mathrm{OR}=2.58$, all $\mathrm{p}<0.01$ ).

Conclusion The BRFSS 2015 data indicated that MS without DM and DM without MS had comparable effects on heart attack, and the odds of risk are doubled than US adults with neither DM nor MS.

\section{BACKGROUND}

Coronary heart disease (CHD) is the leading cause of morbidity and mortality worldwide. CHD alone caused approximately 1 of every 7 deaths in the USA with 366801 deaths due to CHD in 2015. ${ }^{1}$ Each year, around 660000 Americans are estimated to have a new heart
Strengths and limitations of this study

- Behavioral Risk Factor Surveillance System is a routine health-related telephone survey assessing a range of conditions.

- Weighted frequency distributions and summary statistics were used to describe the sample characteristics in each group.

- Limitation: chronic diseases were self-reported by answers.

attack (defined as first hospitalised heart attack or CHD death) and around 305000 Americans have a recurrent attack. Furthermore, an additional 160000 silent heart attacks are estimated to occur each year. ${ }^{2}$

Diabetes mellitus (DM), especially type 2 diabetes, is associated with clustered risk factors for CHD. Among adults with DM, the prevalence of hypertension, hypercholesterolaemia and obesity is ranged $75 \%-85 \%$, $70 \%-80 \%$ and $60 \%-70 \%$, respectively. ${ }^{2-4}$ Patients with DM had higher morbidity and mortality of CHD, including heart attack. In a subgroup analysis of the FRagmin during InStability in Coronary artery disease (FRISC) II trial, patients with diabetes with unstable coronary artery disease had a significantly higher rate of heart attack than non-diabetic patients. $^{5}$

Metabolic syndrome (MS) is a multicomponent risk factor for CHD that includes a cluster of individual cardiometabolic risk factors related to abdominal obesity and insulin resistance. Clinically, MS is a useful entity for communicating the nature of lifestyle-related cardiometabolic risk for both patients and clinicians. ${ }^{2} \mathrm{MS}$ is a risk factor for heart attack in both women and men, from all regions and ethnic groups worldwide. ${ }^{6}$ 
DM and MS are both associated with heart attack. Evidence regarding whether MS without DM has stronger association with heart attack than DM without MS, however, is limited. The ongoing Behavioral Risk Factor Surveillance System (BRFSS) assesses chronic conditions, such as DM, hypertension, hypercholesterolaemia and heart attack. ${ }^{7}$ The objective of the present study was to determine whether the risk of heart attack differs in people with DM without MS and MS without DM using the 2015 BRFSS database.

\section{METHODS}

\section{Participants}

BRFSS is the nation's premier system of health-related telephone surveys that collect state data about US residents regarding their health-related risk behaviours, chronic health conditions and use of preventive services. BRFSS completes more than 400000 adult interviews each year, making it the largest continuously conducted health survey system in the world. ${ }^{8}$ In 2015 , fifty states, the District of Columbia, Guam and Puerto Rico collected data from interviews conducted both by landline telephone and cellular telephone. Questions used in this study in 2015 BRFSS survey include heart attack history, diabetes history, physical activity, dyslipidaemia, hypertension awareness, chronic health conditions, alcohol consumption, fruits and vegetables and currently smoking. ${ }^{9}$

There were 441456 subjects in the 2015 BRFSS survey. The response rate from cellular telephone is $47.2 \%$, which is slightly lower than that from landline telephone $(48.2 \%) .{ }^{10}$ Unknown responses or non-responses were coded as missing in questions included in the study, and there were 332008 subjects included in the analysis after removing missing values.

\section{Measures}

Sociodemographic variables, such as age (18-44years or $45+$ years), race, ethnicity (Hispanic, Latino/Latina, or Spanish origin or no), education, smoking status (current smoker or not) and annual household income, were categorised according to the original variables.

Respondents' lifestyles were assessed by questions on their physical activity, fruit consumption and vegetable consumption. Fruit consumption was categorised as 'consumed fruit one or more times per day' or 'consumed fruit less than one time per day'. Vegetable consumption was categorised as 'consumed vegetables one or more times per day' or 'consumed vegetables one or more times per day'. Physical activity index was categorised as whether 'meet aerobic recommendations' or not.

In the 2015 BRFSS, chronic diseases were self-reported by answers to questions on chronic diseases history. Heart attack was defined as yes to the question, 'Has a doctor, nurse, or other health professional ever told you had a heart attack, also called a myocardial infarction?' Diabetes was defined by a yes answer to the question, 'Has a doctor, nurse, or other health professional ever told you have diabetes?'
Respondents with pre-diabetes, borderline diabetes or gestational diabetes were excluded. Body mass index (BMI) was calculated by self-reported height and weight. Similarly, hypertension was defined as a yes answer to the question, 'Have you ever been told by a doctor, nurse or other health professional that you have high blood pressure?' Borderline hypertension, prehypertension and gestational hypertension were all excluded from the study. Dyslipidaemia was defined as a yes answer to the question, 'Have you ever been told by a doctor, nurse or other health professional that your blood cholesterol is high?' Stroke was defined as yes to the question of 'ever told you had a stroke'. Depression was a yes answer to the question of 'ever told you that you have a depressive disorder, including depression, major depression, dysthymia, or minor'.

MS was diagnosed based on the Adult Treatment Panel III (ATP-III) definition. ${ }^{11}$ The components of MS were abdominal obesity (waist circumference $>40$ inches in men or $>35$ inches in women), triglycerides $\geq 150 \mathrm{mg} / \mathrm{dL}$, high-density lipoprotein cholesterol $<40 \mathrm{mg} / \mathrm{dL}$ in men or $<50 \mathrm{mg} / \mathrm{dL}$ in women, blood pressure $\geq 130 / 85 \mathrm{~mm}$ $\mathrm{Hg}$ and fasting glucose $\geq 110 \mathrm{mg} / \mathrm{dL}$. There were no available data on waist circumference, blood pressure, fasting glucose and lipid profile. The diagnosis of MS was revised based on the questions in the BRFSS. The revised components of MS included diabetes, hypertension, $\mathrm{BMI} \geq 25.0 \mathrm{~kg} / \mathrm{m}^{2}$ and dyslipidaemia. Respondents who had at least three components were regarded as having MS. In this study, the 'MS without DM' group means that respondents had the other three components of MS excluding diabetes.

\section{Statistical analysis}

Each record in the 2015 BRFSS data was weighted using raking weighting methodology. ${ }^{12}$ Raking adjusted the BRFSS data to allow under-represented groups in the sample to be more accurately represented in the final data set. Final weights were assigned to each respondent. All statistical analyses and prevalence estimates have been weighted. Weighted percentages of respondents who ever had heart attack were calculated.

Weighted $\chi^{2}$ tests were performed to determine respondents' characteristic differences across groups. A weighted hierarchical logistic regression was used to examine the difference between the four groups in their association with the risk of a heart attack. ORs and corresponding 95\% CIs were derived from weighted hierarchical logistic regression analysis. Survey-related procedures in SAS V.9.4 (SAS Institute) were used for all data analyses. The significance level was set at $\mathrm{p}<0.05$, and all tests were two sided.

\section{Patient and public involvement}

This study was an analysis of the 2015 BRFSS database. The database was downloaded via the US Centers for Disease Control and Prevention website. 


\section{RESULTS}

\section{Demographic characteristics}

There were 332008 respondents involved in this study. All respondents were categorised into four groups as follows: neither DM nor MS, DM without MS (having DM without MS), MS without DM (having MS without DM) and DM plus MS. There were 237334 respondents with neither DM nor MS, 45191 respondents with DM without MS, 8416 respondents with MS without DM and 41067 respondents with both DM and MS (table 1). Differences in the percentages of gender, age category, smoking status, education level, race, ethnicity and annual household income were statistically significant among the four groups $(p<0.01)$. In addition, the above characteristics were significantly different between DM without MS and MS without DM groups $(\mathrm{p}<0.001)$. In both MS and DM group, $91 \%$ were aged over 45 years, and $21.5 \%$ did not graduate high school, which were higher than the other three groups. Moreover, $17.6 \%$ of respondents in the MS and DM group had annual household incomes lower than $\$ 15000$ and the low income percentage is much higher than the other three groups. Less people were white in the DM without MS group (71.4\%) compared with that in the MS without DM group $(80.4 \%)$. However, more respondents were of Hispanic, Latino, or Spanish origin in the DM without MS group (19.3\%) than in the MS without DM group (10.3\%, $\mathrm{p}<0.001$ ), and more respondents were current smokers in the DM without MS group (16.0\%) compared with the MS without DM group (15.3\%, $\mathrm{p}<0.001$, table 1$)$.

\section{Lifestyle}

Lifestyle measurements were also compared in the four groups (table 1). The percentage of physical activity index, daily fruit consumption and vegetable consumption were all significantly different across the four groups. The physical activity index in the DM without MS and MS without DM groups was $48.2 \%$ and $47.6 \%$, respectively $(\mathrm{p}<0.001)$. The DM and MS group had the least percentage of respondents whose physical activity met the aerobic recommendations. The percentage of respondents who consumed fruit one or more times per day was higher in the DM without MS group, compared with that in the MS without DM group (58.8\% vs $56.8 \%$, $\mathrm{p}<0.001)$. However, daily vegetable consumption was similar between the DM without MS and the MS without DM groups ( $76.9 \%$ vs $76.8 \%, \mathrm{p}=0.019$ ). In the DM and MS group, the percentage of daily vegetable consumption is the least among the four groups (73.4\%).

\section{MS components and chronic diseases}

Among the 332008 respondents, 21896 had heart attack, accounting for the prevalence of $5.2 \%$. MS without DM had higher prevalence of heart attack than that in DM without MS $(11.0 \%$ and $8.5 \%$, respectively, $\mathrm{p}<0.001)$. The prevalence of heart attack in the DM plus MS group was the highest $(16.1 \%$, table 2$)$. The overall prevalence of dyslipidaemia, hypertension, diabetes and $\mathrm{BMI} \geq 25.0 \mathrm{~kg} /$ $\mathrm{m}^{2}$ was $36.6 \%, 37.5 \%, 13.2 \%$ and $67.2 \%$, respectively (table 2). In the DM without MS group, $83 \%$ respondents had one component of MS other than DM, with $17 \%$ people having no other components of MS besides DM.

The overall prevalence of stroke was $3.6 \%$. The prevalence of stroke was significantly different between the DM without MS and MS without DM groups $(4.8 \%$ vs $6.6 \%, \mathrm{p}<0.001)$. The prevalence of stroke in the DM plus MS group was the highest among the four groups $(9.7 \%)$. The overall prevalence of depression was $18.2 \%$. Compared with DM without MS, MS without DM had significantly higher prevalence of depression $(16.4 \%$ vs $24.1 \%, \mathrm{p}<0.001)$. The highest prevalence of depression was observed in the DM plus MS group (27.7\%).

\section{Logistic regression}

Logistic regression was conducted to compare the difference among the four groups in their association with heart attack, using the neither DM nor MS group as the reference (table 3). Results from unadjusted logistic regression analysis showed that both DM without MS $(\mathrm{OR}=3.28$, 95\% CI 2.81 to 3.82) and MS without DM (OR=4.37, 95\% CI 4.06 to 4.70 ) groups had significantly elevated odds of heart attack than neither DM nor MS group. The DM plus MS group had the highest odds of heart attack among the three groups ( $\mathrm{OR}=6.79,95 \%$ CI 6.33 to 7.28$)$.

To identify an independent relationship between DM, MS and heart attack, hierarchical logistic regression analysis was performed. After adjusting for confounders (gender, age, education, smoking, race, physical activity index, daily fruit consumption, daily vegetable consumption, stroke and depression) DM without MS and MS without DM were found to have independently increased odds of heart attack compared with the neither DM nor MS group (DM without MS, adjusted $\mathrm{OR}=2.09$, 95\% CI 1.72 to 2.54; $\mathrm{MS}$ without $\mathrm{DM}$, adjusted $\mathrm{OR}=2.58,95 \% \mathrm{CI}$ 2.36 to 2.81). The DM plus MS group had the highest odds of heart attack (adjusted $\mathrm{OR}=3.45,95 \%$ CI 3.16 to 3.77 , all $\mathrm{p}<0.001$, table 3 ).

\section{DISCUSSION}

In the 2015 BRFSS data, respondents with MS without DM and DM without MS were both associated with elevated risk of heart attack and the amount of increase is doubled compared with respondents with neither DM nor MS. MS did not appear to be a greater odds for heart attack than DM from our analysis results. MS combined with DM increased more risk of heart attack by over 3.4-fold compared with respondents with neither DM nor MS.

MS is a cluster of risk factors contributing to the pathogenesis of atherosclerosis. ${ }^{13}$ There are several definitions of MS and different definitions of MS had different components. ${ }^{11} 1415$ Many large-scale clinical trials and meta-analyses have reported that the presence of MS is a strong predictor for heart attack in many different populations. ${ }^{6}{ }^{16-18}$ In the INTERHEART case-control study involving 26903 subjects from 52 countries, MS was associated with an increased risk of heart attack, both using 


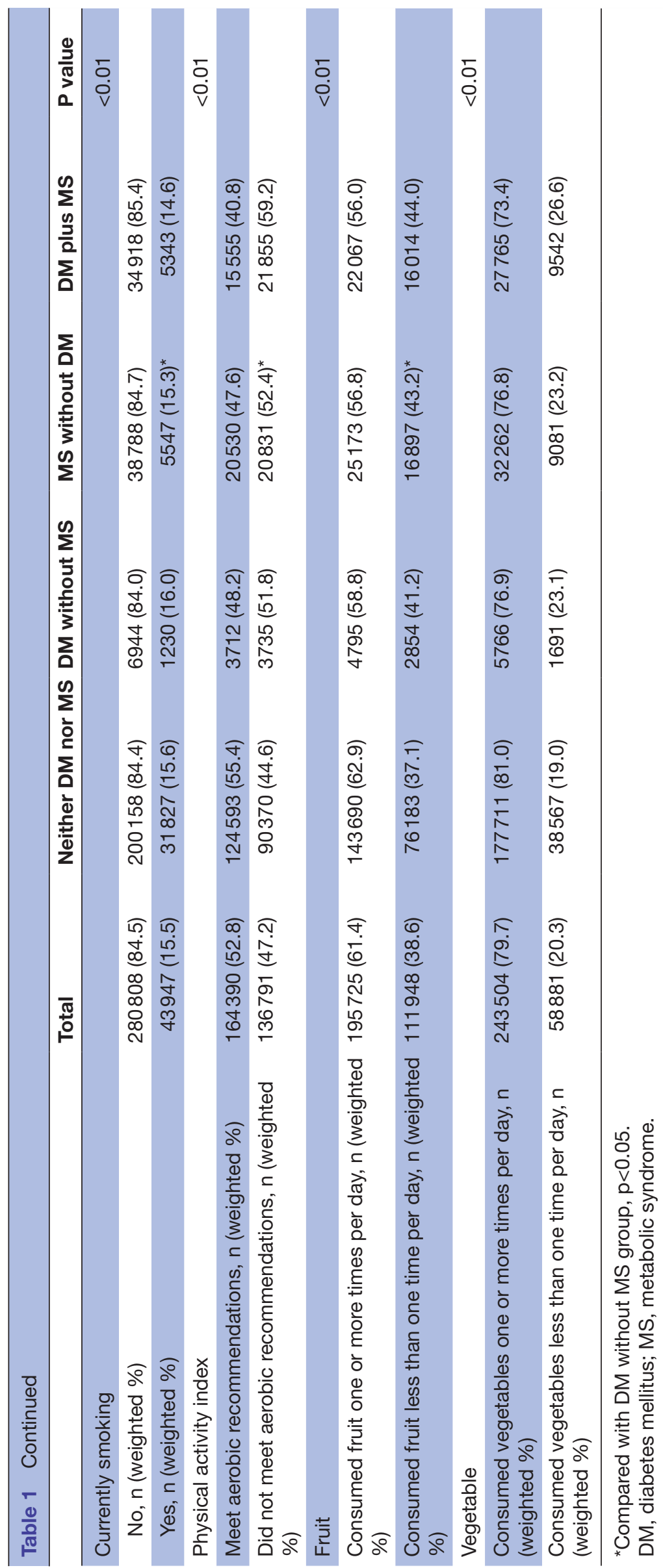


Table 2 Chronic diseases among the four groups according to the presence of metabolic syndrome and diabetes

\begin{tabular}{|c|c|c|c|c|c|c|}
\hline Chronic diseases & Total & $\begin{array}{l}\text { Neither DM nor } \\
\text { MS }\end{array}$ & DM without MS & MS without DM & DM plus MS & $P$ value \\
\hline Heart attack, $\mathrm{n}$ (weighted \%) & $21896(5.2)$ & $8863(2.7)$ & $851(8.5)$ & $5310(11.0)^{*}$ & $6872(16.1)$ & $<0.01$ \\
\hline Dyslipidaemia, n (weighted \%) & $140653(36.6)$ & $62526(22.2)$ & $1102(12.2)$ & $45191(100.0)^{*}$ & $31834(77.6)$ & $<0.01$ \\
\hline $\mathrm{BMl} \geq 25.0 \mathrm{~kg} / \mathrm{m}^{2}, \mathrm{n}$ (weighted \%) & $223112(67.2)$ & $135589(59.1)$ & $4551(56.8)$ & $45191(100.0)^{*}$ & 37781 (92.3) & $<0.01$ \\
\hline Stroke, $\mathrm{n}$ (weighted \%) & $15013(3.6)$ & $6910(2.2)$ & $544(4.8)$ & $3228(6.6)^{*}$ & $4331(9.7)$ & $<0.01$ \\
\hline
\end{tabular}

${ }^{*}$ Compared with DM without MS group, $\mathrm{p}<0.05$.

BMI, body mass index; DM, diabetes mellitus; MS, metabolic syndrome.

the WHO definition $(\mathrm{OR}=2.69)$ and the International Diabetes Federation (IDF) definition $(\mathrm{OR}=2.20)$.The direction of associations was similar across all regions and ethnic groups. ${ }^{6}$ A large family study in Finland and Sweden of 4483 subjects also identified the association

Table 3 The OR and $95 \% \mathrm{Cls}$ of DM and MS related to heart attack in the hierarchy logistic regression analysis

\begin{tabular}{|c|c|c|c|}
\hline & OR & $95 \% \mathrm{Cl}$ & P value \\
\hline \multicolumn{4}{|l|}{$\begin{array}{l}\text { Model } 1 \\
(n=332008)\end{array}$} \\
\hline DM without MS & 3.28 & 2.81 to 3.82 & $<0.01$ \\
\hline MS without DM & 4.37 & 4.06 to 4.70 & $<0.01$ \\
\hline DM plus MS & 6.79 & 6.33 to 7.28 & $<0.01$ \\
\hline \multicolumn{4}{|l|}{$\begin{array}{l}\text { Model } 2 \\
(n=319712)\end{array}$} \\
\hline DM without MS & 2.10 & 1.77 to 2.49 & $<0.01$ \\
\hline MS without DM & 2.85 & 2.64 to 3.09 & $<0.01$ \\
\hline DM plus MS & 4.06 & 3.76 to 4.38 & $<0.01$ \\
\hline \multicolumn{4}{|l|}{$\begin{array}{l}\text { Model } 3 \\
(n=282332)\end{array}$} \\
\hline DM without MS & 2.12 & 1.75 to 2.56 & $<0.01$ \\
\hline MS without DM & 2.82 & 2.59 to 3.07 & $<0.01$ \\
\hline DM plus MS & 3.99 & 3.66 to 4.34 & $<0.01$ \\
\hline \multicolumn{4}{|l|}{$\begin{array}{l}\text { Model } 4 \\
(n=280977)\end{array}$} \\
\hline DM without MS & 2.09 & 1.72 to 2.54 & $<0.01$ \\
\hline MS without DM & 2.58 & 2.36 to 2.81 & $<0.01$ \\
\hline DM plus MS & 3.45 & 3.16 to 3.77 & $<0.01$ \\
\hline
\end{tabular}

Model 1: unadjusted.

Model 2: adjusted for gender, age (45 years or not), education, current smoking, race.

Model 3: adjusted for gender, age (45 years or not), education, current smoking, race, physical activity index, fruits consumed one or more times per day, vegetable consumed one or more times per day.

Model 4: adjusted for gender, age (45years or not), education, current smoking, race, physical activity index, fruits consumed one or more times per day, vegetable consumed one or more times per day, stroke and depression.

DM, diabetes mellitus; MS, metabolic syndrome. between MS and an increased risk of heart attack in all subjects using the WHO definition. ${ }^{18}$ Similar results were observed when the 2001 National Cholesterol Education Program (NCEP) and 2004 revised NCEP definitions were used. ${ }^{16}{ }^{17}$ In our analysis, the association between MS and heart attack was consistent. MS, regardless of its definition, was associated with heart attack.

$\mathrm{DM}$ is one of the components in most definitions of MS. The risk for cardiovascular disease (CVD) is twofold to eightfold higher in the diabetic population than that in the non-diabetic population of a similar age, sex and ethnicity, and CVD is the leading cause of morbidity and mortality among patients with type 2 diabetes. ${ }^{19-21}$

Previous researchers have investigated the effects of DM on heart attack. Consistent with our findings, it has been reported that DM was associated with an increased heart attack risk in both men and women. ${ }^{22} \mathrm{~A}$ cohort study using the UK General Practice Research Database showed a much larger relative risk of heart attack in DM. ${ }^{23}$

Both DM and MS were associated with an increased risk of heart attack. However, evidence regarding whether MS without DM is better than DM without MS for evaluating heart attack is limited. There were studies to evaluate the relationship between MS and DM on CVD events. Results from different studies regarding differences in CVD events between DM and MS were conflicting. The Ansung-Ansan cohort study showed there was no difference in the risk of incident CVD between individuals with DM without MS and MS without DM. ${ }^{24}$ Yet, in the REduction of Atherothrombosis for Continued Health (REACH) registry, presence of newly detected DM but not MS was associated with an increased risk of CVD events. ${ }^{25}$ Besides the difference in population characteristics in these studies, the sample size and the definitions of CVD may affect the results.

There were fewer studies conducted in US adults to compare the effects of MS and DM on heart attack. In the logistic analysis of this study, MS without DM and DM without MS were found to have similar odds of heart attack. This showed that MS and DM may have similar effects on heart attack in the US adults, which was different from the results of previous study in US population. ${ }^{26}$ Our results indicated that to prevent heart attack or CVD, even a diabetic person does not meet the criteria 
of MS, much more attention should be paid to control metabolic abnormalities.

DM typically copresents with at least one metabolic abnormality. In our analysis, the weighted prevalence of hypertension, dyslipidaemia and overweight in DM without MS group was $13.9 \%, 12.2 \%$ and $56.8 \%$, respectively. Of the respondents with DM, $83 \%$ had at least one or more components of MS other than DM. As shown in a population-based cohort study, DM with only one component of MS had more than twofold higher CVD risk than those with DM only. ${ }^{27}$ These associations may be helpful to explain in this study why DM and MS had similar effects on heart attack. Further studies were needed to evaluate the association between MS without DM and DM without MS with heart attack

There were some limitations in our study. First, the definition of MS was revised according to the contents of 2015 BRFSS. MS was diagnosed based on the ATP-III definition. ${ }^{11}$ The components of MS were diabetes, hypertension, $B M I \geq 25.0 \mathrm{~kg} / \mathrm{m}^{2}$ and dyslipidaemia. Respondents who had at least three components were regarded as having MS. According to the ATP-III definition, central obesity was diagnosed based on waist circumference. We used $\mathrm{BMI} \geq 25.0 \mathrm{~kg} / \mathrm{m}^{2}$ to classify individuals because waist circumference was not available. The MS definition from the American College of Endocrinology recommends that $\mathrm{BMI}>25 \mathrm{~kg} / \mathrm{m}^{2}$ or a waist circumference $>40$ inches for men, $>35$ inches for women was regarded as obesity. ${ }^{28}$ Therefore, in the present study, we used BMI $\geq 25 \mathrm{~kg} / \mathrm{m}^{2}$ as a component of MS. Second, in the 2015 BRFSS, there were no data on triglyceride and high-density lipoprotein. Dyslipidaemia was assessed by whether respondents had ever been told their blood cholesterol was high. Third, the self-reported nature of the cross-sectional study may lead to underestimate the actual prevalence of heart attack. In this study, $13.2 \%$ of respondents had diabetes. However, some diabetic respondents may have silent heart attack without any symptoms. In the BRFSS survey the data of fatal heart attack are not included, which may also underestimate the actual prevalence of heart attack. Fourth, gestational diabetes and pre-diabetes were excluded. These two conditions are both important risk factors for DM that has been excluded from the study. In this study, $24.8 \%$ of subjects in the 2015 BRFSS data with unknown responses or non-responses in questions included in the study were excluded from the analysis under the assumption of missing completely at random, which might result in some bias of the results when the assumption is not valid.

In conclusion, even though the weighted percentage of heart attack in MS without DM was higher than that in DM without MS, MS and DM had similar effects on heart attack, which could double the risk of heart attack. Furthermore, when MS is combined with DM, the risk of heart attack will be increased by over 3.4-fold. Considering the nature of the cross-sectional study in the 2015 BRFSS data, prospective studies are needed to confirm the association between MS without DM and DM without MS with heart attack.
Contributors GRY and DL designed the study and analysed the data. GRY drafted the manuscript. DL and TDD revised the manuscript. All authors read and approved the final manuscript.

Funding This work was supported by Beijing Municipal Training Foundation for Highly Qualified and Technological Talents of Health System (2014-3-013) and Capital's Funds for Health Improvement and Research (2016-2-2054). DL and TDD's time is partly supported by the University of Rochester's Clinical and Translational Science Award (CTSA) (numbers UL1 TR000042 and UL1 TR002001) from the National Center for Advancing Translational Sciences of the National Institutes of Health.

Competing interests None declared.

Patient consent for publication Not required.

Ethics approval The 2015 BRFSS annual survey data do not include any identifiable information and are publicly available from the Centers for Disease Control and Prevention website (https://www.cdc.gov/brfss/annual_data/annual_ 2015.html).

Provenance and peer review Not commissioned; externally peer reviewed.

Data availability statement All the data are publicly available from the Centers for Disease Control and Prevention website (https://www.cdc.gov/brfss/annual_data/ annual_2015.html).

Open access This is an open access article distributed in accordance with the Creative Commons Attribution Non Commercial (CC BY-NC 4.0) license, which permits others to distribute, remix, adapt, build upon this work non-commercially, and license their derivative works on different terms, provided the original work is properly cited, appropriate credit is given, any changes made indicated, and the use is non-commercial. See: http://creativecommons.org/licenses/by-nc/4.0/.

\section{REFERENCES}

1. Benjamin EJ, Virani SS, Callaway CW, et al. Heart disease and stroke Statistics-2018 update: a report from the American heart association. Circulation 2018;137:e67-492.

2. Mozaffarian D, Benjamin EJ, Go AS, et al. Heart disease and stroke Statistics-2016 update: a report from the American heart association. Circulation 2016;133:e38-60.

3. Selvin E, Parrinello CM, Sacks DB, et al. Trends in prevalence and control of diabetes in the United States, 1988-1994 and 1999-2010. Ann Intern Med 2014;160:517-25.

4. Preis SR, Pencina MJ, Hwang S-J, et al. Trends in cardiovascular disease risk factors in individuals with and without diabetes mellitus in the Framingham heart study. Circulation 2009;120:212-20.

5. Norhammar A, Malmberg K, Diderholm E, et al. Diabetes mellitus: the major risk factor in unstable coronary artery disease even after consideration of the extent of coronary artery disease and benefits of revascularization. J Am Coll Cardiol 2004;43:585-91.

6. Mente A, Yusuf S, Islam S, et al. Metabolic syndrome and risk of acute myocardial infarction a case-control study of 26,903 subjects from 52 countries. J Am Coll Cardiol 2010;55:2390-8.

7. Mokdad AH, Stroup DF, Giles WH, et al. Public health surveillance for behavioral risk factors in a changing environment. recommendations from the behavioral risk factor surveillance team. MMWR Recomm Rep 2003;52:1-12.

8. Centers for Disease Control and Prevention. Behavioral risk factor surveillance system (BRFSS). About BRFSS. Available: https://www. cdc.gov/brfss/about/indexhtm [Accessed 30 March 2017].

9. 2015 BRFSS overview. Available: https://www.cdc.gov/brfss/annual_ data/2015/pdf/overview_2015pdf [Accessed 30 March 2017].

10. 2015 summary data quality report with response rates. Available: https://www.cdc.gov/brfss/annual_data/2015/pdf/2015-sdqrpdf [Accessed on 30 March 2017].

11. Expert Panel on Detection, Evaluation, and Treatment of High Blood Cholesterol in Adults. Executive summary of the third report of the National cholesterol education program (NCEP) expert panel on detection, evaluation, and treatment of high blood cholesterol in adults (adult treatment panel III). JAMA 2001;285:2486-97.

12. Centers for Disease Control and Prevention. Behavioral risk factor surveillance system. Weighting BRFSS data BRFSS, 2015. Available: https://www.cdc.gov/brfss/annual_data/2015/pdf/weighting_thedata_webpage_contentpdf [Accessed 30 March 2017].

13. Al-Aqeedi RF, Abdullatef WK, Dabdoob W, et al. The prevalence of metabolic syndrome components, individually and in combination, in male patients admitted with acute coronary syndrome, without previous diagnosis of diabetes mellitus. Libyan J Med 2013;8:20185. 
14. Alberti KG, Zimmet PZ, Definition ZPZ. Definition, diagnosis and classification of diabetes mellitus and its complications. Part 1 : diagnosis and classification of diabetes mellitus provisional report of a who consultation. Diabet Med 1998;15:539-53.

15. Alberti KGMM, Zimmet P, Shaw J, et al. The metabolic syndrome--a new worldwide definition. Lancet 2005;366:1059-62.

16. Mottillo S, Filion KB, Genest J, et al. The metabolic syndrome and cardiovascular risk a systematic review and meta-analysis. J Am Coll Cardiol 2010;56:1113-32.

17. Shin J-A, Lee J-H, Lim S-Y, et al. Metabolic syndrome as a predictor of type 2 diabetes, and its clinical interpretations and usefulness. $J$ Diabetes Investig 2013;4:334-43.

18. Isomaa B, Almgren P, Tuomi T, et al. Cardiovascular morbidity and mortality associated with the metabolic syndrome. Diabetes Care 2001;24:683-9.

19. Papa G, Degano C, lurato MP, et al. Macrovascular complication phenotypes in type 2 diabetic patients. Cardiovasc Diabetol 2013;12:20.

20. Haffner SM, Lehto S, Rönnemaa T, et al. Mortality from coronary heart disease in subjects with type 2 diabetes and in nondiabetic subjects with and without prior myocardial infarction. N Engl J Med 1998;339:229-34.

21. Brun E, Nelson RG, Bennett PH, et al. Diabetes duration and cause-specific mortality in the Verona diabetes study. Diabetes Care 2000;23:1119-23.
22. Laugsand LE, Janszky I, Vatten LJ, et al. Autoimmune diabetes in adults and risk of myocardial infarction: the HUNT study in Norway. $J$ Intern Med 2016;280:518-31.

23. Mulnier HE, Seaman HE, Raleigh VS, et al. Risk of myocardial infarction in men and women with type 2 diabetes in the UK: a cohort study using the general practice research database. Diabetologia 2008;51:1639-45.

24. Bae JC, Cho NH, Suh S, et al. Cardiovascular disease incidence, mortality and case fatality related to diabetes and metabolic syndrome: a community-based prospective study (Ansung-Ansan cohort 2001-12). J Diabetes 2015;7:791-9.

25. Udell JA, Steg PG, Scirica BM, et al. Metabolic syndrome, diabetes mellitus, or both and cardiovascular risk in outpatients with or at risk for atherothrombosis. Eur J Prev Cardiol 2014;21:1531-40.

26. Church TS, Thompson AM, Katzmarzyk PT, et al. Metabolic syndrome and diabetes, alone and in combination, as predictors of cardiovascular disease mortality among men. Diabetes Care 2009;32:1289-94.

27. Bruno G, Merletti F, Biggeri A, et al. Metabolic syndrome as a predictor of all-cause and cardiovascular mortality in type 2 diabetes: the Casale Monferrato study. Diabetes Care 2004;27:2689-94.

28. Einhorn D, Reaven GM, Cobin RH, et al. American College of endocrinology position statement on the insulin resistance syndrome. Endocr Pract 2003;9:237-52. 